\title{
The Relationship of Lactate Levels with Carboxyhemoglobin Levels and Clinical Findings in Patients Admitted with Acute Carbon Monoxide Poisoning
}

\author{
Akut Karbon Monoksit Zehirlenmesiyle Başvuran Hastaların Laktat Düzeyinin \\ Karboksihemoglobin ve Klinik Bulgularla İlişkisi
}

Eren USUL ${ }^{1}$

(D) 0000-0003-3980-6768

Ali HALICI ${ }^{2}$

(D) 0000-0003-1392-4694

Mehmet Hilmi HÖKE

(D) 0000-0002-3905-9849

${ }^{1}$ Dışkapı Yıldırım Beyazıt Training and Research Hospital Department of Emergency Medicine, Ankara, Turkey ${ }^{2}$ Polatl Duatepe State Hospital Emergency Medicine, Ankara, Turkey ${ }^{3}$ Sincan Dr. Nafiz Körez State Hospital Emergency Medicine, Ankara, Turkey

\section{Corresponding Author Sorumlu Yazar \\ Eren USUL \\ usuleren7@hotmail.com}

Received / Geliş Tarihi : 22.12.2020 Accepted / Kabul Tarihi : 11.03.2021 Available Online /

Çevrimiçi Yayın Tarihi : 22.03.2021

\begin{abstract}
Aim: Acute carbon monoxide (CO) poisoning is a potentially mortal, though preventable, condition. Mild poisoning presents with non-specific symptoms, such as fatigue, headache, nausea and vomiting, whereas severe exposure to $\mathrm{CO}$ can result in loss of consciousness, coma, and death. The aim of this study was to investigate the utility of lactate and carboxyhemoglobin $(\mathrm{COHb})$ levels in the clinical presentation and treatment of patients with acute $\mathrm{CO}$ poisoning. Material and Methods: Data were obtained from the hospital information system and patient files with ICD-10 code "T58: Toxic effects of CO". The blood parameters and vital signs of patients at admission, causes of poisoning, time to hospital, and Glasgow coma scores during admission were recorded. Within related and relevant complications of $\mathrm{CO}$ intoxication and data concerning treatment plans and hospitalization status were recorded.

Results: A statistically significant difference was found between $\mathrm{COHb}$ and lactate levels of patients who did and did not develop neurological and cardiac complications $(p<0.001)$. Moreover, a statistically significant difference was found in $\mathrm{COHb}$ and lactate levels among patients who did or did not receive hyperbaric oxygen therapy, and who were hospitalized or not $(\mathrm{p}<0.001)$. As looking for biochemical profile, significant correlations was found between $\mathrm{COHb}$ and $\mathrm{pH}$, base excess, and bicarbonate and lactate levels.

Conclusion: This study shows that $\mathrm{COHb}$ and lactate levels at admission to the emergency department are significant for prognosis, follow-up, and treatment of patients with $\mathrm{CO}$ poisoning. Elevated lactate and $\mathrm{COHb}$ levels may also found to be associated with neurological and cardiac complications.

Keywords: Poisoning; carbon monoxide; lactate; hyperbaric oxygenation; carboxyhemoglobin.
\end{abstract}

ÖZ

Amaç: Akut karbon monoksit (carbon monoxide, CO) zehirlenmesi, ölümle sonuçlanan önemli ve önlenebilir bir zehirlenme nedenidir. Hafif zehirlenmelerde yorgunluk, baş ağrısı, bulantı ve kusma gibi spesifik olmayan belirtiler görülürken şiddetli maruziyetlerde bilinç kaybı, koma ve ölüm ortaya çıkabilir. Bu çalışmanın amacı, laktat ve karboksihemoglobin (carboxyhemoglobin, $\mathrm{COHb}$ ) düzeylerinin akut $\mathrm{CO}$ zehirlenmesi olan hastaların klinik ve tedavi sürecindeki yerini araştırmaktır.

Gereç ve Yöntemler: Veriler hastane otomasyon sistemi ve hasta dosyalarından, ICD-10 kodu "T58: Karbonmonoksitin Toksik Etkisi" olanlar seçilerek elde edildi. Hastaların başvuru sırasındaki kan parametreleri ve yaşamsal belirtileri, zehirlenme nedenleri, hastaneye geliş süreleri ve başvuru sırasındaki Glasgow koma skorları kayıt altına alınmıştır. Hastaların CO zehirlenmesine bağlı ve zehirlenme ile ilgili komplikasyonları ve aldıkları tedavi planı ve hastaneye yatış durumu ile ilgili verileri kaydedilmiştir.

Bulgular: Nörolojik ve kardiyak komplikasyon görülen ve görülmeyen hastalar arasında CO ve laktat değerlerinde istatistiksel olarak anlamlı bir fark bulunmuştur $(p<0,001)$. Ayrica hiperbarik oksijen tedavisi alan ve almayan hastalar ile hastaneye yatışı olan ve olmayan hastalar arasında $\mathrm{COHb}$ ve laktat değerlerinde istatistiksel olarak anlamlı bir farklılık bulunmuştur $(\mathrm{p}<0,001)$. Biyokimyasal parametreler açısından bakıldığında, $\mathrm{COHb}$ ile $\mathrm{pH}$, baz açı̆̆ı, bikarbonat ve laktat düzeyleri arasında anlamlı korelasyon saptanmıştır.

Sonuç: $\mathrm{Bu}$ çalışmada, acil servise başvuru anındaki $\mathrm{COHb}$ ve laktat değerlerinin, $\mathrm{CO}$ zehirlenmesi olan hastaların prognozu, klinik seyrinin takip edilmesi ve tedavi sürecinde önemli olduğu gösterilmiştir. Ayrıca, yüksek laktat ve $\mathrm{COHb}$ değerlerinin nörolojik ve kardiyak komplikasyon gözlenmesiyle de ilişkili olabileceği düşünülmektedir.

Anahtar kelimeler: Zehirlenme; karbon monoksit; laktat; hiperbarik oksijen; karboksihemoglobin. 


\section{INTRODUCTION}

Acute carbon monoxide (CO) poisoning is a prominent and potentially fatal, however preventable condition and is a leading cause of accidental poisoning. It is a serious public health problem in developing countries due to the widespread use of coal stoves and various other hazardous heating systems (1-7). Mild intoxications may present with non-specific symptoms such as fatigue, headache, nausea, and vomiting, whereas severe exposure to $\mathrm{CO}$ can result in loss of consciousness, coma, and death (2).

Carbon monoxide is released into the environment via the incomplete combustion of carbon-based compounds (8). It is a tasteless, odorless, and colorless gas, often causing patients to lose consciousness before realizing they are poisoned. Decreased oxygen supply results in hypoxemia (9). Due to their high metabolic requirement, the heart and brain are the chiefly sensitive to $\mathrm{CO}$ poisoning, and therefore death and neurological sequelae are the most common and devastating complications of this intoxication $(10,11)$.

Lactate, a by-product of anaerobic glycolysis, is an important indicator of tissue hypoxia, and high lactate levels are increasingly utilized in the treatment of critical patients with CO poisoning (12-14). In addition, a study on adults found that the use of lactate levels recorded at admission, along with clinical symptoms and carboxyhemoglobin $(\mathrm{COHb})$ levels, can be a useful predictor of intoxication severity (7). In this study, the aim was to analyze the utility of blood lactate and $\mathrm{COHb}$ levels in the interpretation of clinical presentation and treatment of patients with acute $\mathrm{CO}$ intoxication.

\section{MATERIALS AND METHODS}

This was a retrospective cohort study and conducted in compliance with the principles of the World Medical Association Declaration of Helsinki and in accordance with research guidelines. This study was approved by the Ethics Committee of Ankara Dışkapı Training and Research Hospital (16.02.2015, 20/04). The inclusion criteria were: 1) being over 18 years of age and 2) having a diagnosis of acute $\mathrm{CO}$ poisoning. The exclusion criteria were: 1) pregnancy, and 2) being below 18 years of age. Data were collected in the tertiary education and research hospital emergency room between 2016 and 2017.

Data were recorded from the hospital information system and patient files with the ICD-10 code T58. The analyzed laboratory parameters included hemogram, blood biochemical profile, bleeding profile, and blood gas results. The blood results and vital signs of the patients at admission, their cause of poisoning, the time to hospital, Glasgow coma scores (GCS) at admission, relevant neurological (loss of consciousness, syncope, fainting) and cardiac complications (myocardial ischemia and/or ECG changes), and data concerning treatment and their status of hospitalization were recorded.

At the clinic, the criteria for receiving hyperbaric oxygen (HBO) therapy comprised of altered consciousness, ischemic ECG changes, focal neurological deficits, pregnant women with $\mathrm{COHb}>15 \%$, and patients with headache and nausea together with a COHb level $>25 \%$.

\section{Statistical Analysis}

Data were analyzed using IBM SPSS Statistics v.25.0 (IBM Corp., Armonk, NY, USA) and MedCalc v.15.8 (MedCalc Software bvba, Ostend, Belgium) package. The data were summarized using descriptive statistics (frequency, percentage, mean, standard deviation, median and $\min -\max$ ), and the qualitative data were evaluated using the Pearson chi-square test. The conformity of data to normal distribution was evaluated with the Kolmogorov-Smirnov test. Quantitative data showing normal distribution was evaluated using the Independent Samples $t$ test, and the Mann-Whitney U test was used for numerical data that did not conform to the normal distribution. Correlations between the variables were evaluated using Pearson's and Spearman's correlation coefficient. The receiver operating characteristic (ROC) curve method was used to determine the diagnostic significance of the tested parameters. Cut-off values for the test were set according to Youden's index. Statistical significance level was considered as 0.05 .

\section{RESULTS}

The study included 292 patients comprising 189 (64.7\%) females and $103(35.3 \%)$ males. The mean age was $42.5 \pm 16.9$ (range, 2-94) years. The most common cause of $\mathrm{CO}$ poisoning was coal stoves $(44.2 \%)$. The mean lactate level was $2.4 \pm 1.8 \mathrm{mmol} / \mathrm{L}$ (Table 1 ).

Table 1. Characteristic of patients included in the study

\begin{tabular}{|c|c|c|}
\hline & $\mathbf{n}$ & $\%$ \\
\hline \multicolumn{3}{|l|}{ Gender } \\
\hline Female & 189 & 64.7 \\
\hline Male & 103 & 35.3 \\
\hline \multicolumn{3}{|l|}{ Cause of poisoning } \\
\hline Coal stove & 129 & 44.2 \\
\hline Water heater & 40 & 13.7 \\
\hline \multirow[t]{2}{*}{ Boiler } & 123 & 42.1 \\
\hline & $\operatorname{Mean} \pm$ SD & Median (min-max) \\
\hline Age (years) & $42.5 \pm 16.9$ & $39.5(2.0-94.0)$ \\
\hline $\mathrm{pH}$ & $7.4 \pm 0.1$ & $7.4(6.9-7.6)$ \\
\hline $\mathrm{COHb}(\%)$ & $20.9 \pm 10.9$ & $21.2(0.4-48.8)$ \\
\hline Lactate $(\mathrm{mmol} / \mathrm{L})$ & $2.4 \pm 1.8$ & $2.0(0.2-13.7)$ \\
\hline $\mathrm{BE}(\mathrm{mmol} / \mathrm{L})$ & $-0.7 \pm 3.6$ & $-0.4(-25.7-6.5)$ \\
\hline $\mathrm{HCO}_{3}(\mathrm{mmol} / \mathrm{L})$ & $23.4 \pm 3.1$ & $23.6(7.0-30.6)$ \\
\hline TROP $(\mu \mathrm{g} / \mathrm{L})$ & $0.088 \pm 0.803$ & $0.003(0.0-12.0)$ \\
\hline $\mathrm{CK}(\mathrm{u} / \mathrm{L})$ & $129.2 \pm 152.8$ & $88.5(0.6-1400.0)$ \\
\hline CKMB (IU/L) & $20.5 \pm 19.7$ & $15.5(0.3-202.0)$ \\
\hline $\mathrm{HB}(\mathrm{g} / \mathrm{dL})$ & $13.7 \pm 1.9$ & $13.8(2.5-19.8)$ \\
\hline $\mathrm{WBC}(/ \mu \mathrm{L})$ & $9.2 \pm 4.9$ & $8.4(2.6-65.9)$ \\
\hline $\operatorname{PLT}(/ \mu \mathrm{L})$ & $247.7 \pm 79.3$ & $248.5(17.0-513.0)$ \\
\hline Time to admission (minutes) & $339.8 \pm 253.9$ & $250.0(20.0-1159.0)$ \\
\hline $\mathrm{SAP}(\mathrm{mmHg})$ & $124.6 \pm 22.9$ & $120.0(80.0-220.0)$ \\
\hline DAP $(\mathrm{mmHg})$ & $72.8 \pm 12.4$ & $70.0(50.0-117.0)$ \\
\hline Respiratory rate (breaths/min) & $17.5 \pm 3.2$ & $18.0(10.0-26.0)$ \\
\hline Temperature $\left({ }^{0} \mathrm{C}\right)$ & $36.4 \pm 0.4$ & $36.4(35.0-38.1)$ \\
\hline Heart rate (bpm) & $88.0 \pm 16.1$ & $84.0(54.0-157.0)$ \\
\hline $\mathrm{sPO}_{2}(\%)$ & $96.1 \pm 4.0$ & $97.0(60.0-100.0)$ \\
\hline GCS & $14.9 \pm 0.5$ & $15.0(9.0-15.0)$ \\
\hline
\end{tabular}

SD: standard deviation, $\mathrm{COHb}$ : carboxyhemoglobin, BE: base excess, $\mathrm{HCO} 3$ : bicarbonate, TROP: Troponin, CK: creatine kinase, CKMB: creatine kinase myocardial band, HB: hemoglobin, WBC: white blood cell count, PLT: platelet count, SAP: systolic arterial pressure, DAP: diastolic arterial pressure, SPO2: oxygen saturation, GCS: Glasgow coma scale 
Comparing the neurological complications with the obtained blood parameters, a difference of statistical significance was found between the patients with and without neurological complications in $\mathrm{COHb}(\mathrm{p}<0.001)$, lactate levels $(\mathrm{p}=0.003)$, base excess $(\mathrm{p}=0.009)$, troponin $(\mathrm{p}=0.002)$, white blood cell $(\mathrm{WBC})$ count $(\mathrm{p}=0.028)$, systolic blood pressure $(\mathrm{p}=0.031)$, and respiratory rate $(\mathrm{p}=0.041)$. No difference of significance was observed in terms of remaining parameters such as the cause of poisoning and gender between the patients with and without neurological complications (Table 2).

Patients with elevated lactate levels were significantly more likely to have cardiac complications and a difference of statistical significance was found between the patients with and without cardiac complications in terms of lactate levels $(\mathrm{p}=0.040)$.

Furthermore, a statistically significant difference was found between patients who did and did not receive $\mathrm{HBO}$ therapy regarding their $\mathrm{COHb}(\mathrm{p}=0.004)$, lactate levels $(\mathrm{p}=0.005)$, base excess $(\mathrm{p}=0.034)$, and systolic blood pressure $(\mathrm{p}=0.031$, Table 3$)$.

Analyzing the biochemical blood parameters of patients admitted with $\mathrm{CO}$ intoxication, a correlation was found between $\mathrm{COHb}$ and $\mathrm{pH}$, base excess, bicarbonate, hemoglobin (HB), and lactate levels. Also, lactate was found to be correlated with all parameters, but more significantly correlated with $\mathrm{pH}(\mathrm{r}=-0.367, \mathrm{p}<0.001)$, base excess $\quad(\mathrm{r}=-0.554, \mathrm{p}<0.001)$, bicarbonate $(\mathrm{r}=-0.544$, $\mathrm{p}<0.001)$, troponin $(\mathrm{r}=0.218, \mathrm{p}<0.001)$, and WBC levels ( $\mathrm{r}=0.219, \mathrm{p}<0.001$, Table 4).

A ROC analysis regarding neurological complications seen in $\mathrm{CO}$ intoxication revealed an area under curve (AUC) of 0.815 (95\% CI, 0.766-0.858) with a cut-off value of 21.2 (sensitivity of $88.7 \%$, specificity of $56.1 \%$ ) for $\mathrm{COHb}$, and an AUC of 0.629 (95\% CI, 0.571-0.685) with a cut-off value of 2.48 (sensitivity of $50.9 \%$, specificity of $75.4 \%$ ) for lactate (Table 5).

Another ROC analysis performed regarding hyperbaric therapy showed an AUC of 0.682 (95\% CI, 0.625-0.735) and cut-off value of 21.8 for $\mathrm{COHb}$ (sensitivity of $75.0 \%$, specificity of $55.6 \%$ ), while for lactate the AUC was 0.710 (95\% CI, 0.654-0.762) and cut-off value was 2.54 (sensitivity of $62.5 \%$, specificity of $74.7 \%$, Table 6).

It was also found that patients who had elevated lactate levels due to carbon monoxide intoxication were significantly more likely to require hospitalization.

Table 2. Comparison of patient characteristics according to neurological complications

\begin{tabular}{|c|c|c|c|}
\hline & Without NC (n=239) & With NC $(n=53)$ & $\mathbf{p}$ \\
\hline \multicolumn{4}{|l|}{ Gender } \\
\hline Female & $154(64.4 \%)$ & $35(66.0 \%)$ & \multirow{2}{*}{$0.951^{\mathrm{a}}$} \\
\hline Male & $85(35.6 \%)$ & $18(34.0 \%)$ & \\
\hline \multicolumn{4}{|l|}{ Cause of poisoning } \\
\hline Coal stove & $99(41.4 \%)$ & $30(56.6 \%)$ & \multirow{3}{*}{$0.130^{\mathrm{a}}$} \\
\hline Water heater & $34(14.2 \%)$ & $6(11.3 \%)$ & \\
\hline Boiler & $106(44.4 \%)$ & $17(32.1 \%)$ & \\
\hline Age (years) & $42.3 \pm 16.6$ & $43.8 \pm 18.3$ & $0.557^{\mathrm{b}}$ \\
\hline $\mathrm{pH}$ & $7.4(7.4-7.4)[7.2-7.6]$ & $7.4(7.3-7.4)[6.9-7.5]$ & $0.105^{\mathrm{c}}$ \\
\hline $\mathrm{COHb}(\%)$ & $19.7 \pm 10.5$ & $25.9 \pm 11.1$ & $<0.001^{\mathrm{b}}$ \\
\hline Lactate $(\mathrm{mmol} / \mathrm{L})$ & $1.9(1.4-2.5)[0.2-11.0]$ & $2.5(1.7-4.1)[0.7-13.7]$ & $\mathbf{0 . 0 0 3}^{\mathrm{c}}$ \\
\hline $\mathrm{BE}(\mathrm{mmol} / \mathrm{L})$ & $-0.26 \pm 2.35$ & $-2.75 \pm 6.62$ & $0.009^{b}$ \\
\hline $\mathrm{HCO}_{3}(\mathrm{mmol} / \mathrm{L})$ & $23.7 \pm 2.5$ & $22.1 \pm 4.9$ & $0.021^{b}$ \\
\hline TROP $(\mu \mathrm{g} / \mathrm{L})$ & $0.0(0.0-0.0)[0.0-0.5]$ & $0.0(0.0-0.1)[0.0-12.0]$ & $0.002^{c}$ \\
\hline $\mathrm{CK}(\mathrm{u} / \mathrm{L})$ & $88.0(60.3-135.8)[0.6-1.400 .0]$ & $95.0(73.3-140.0)[32.0-1.314 .0]$ & $0.400^{c}$ \\
\hline CKMB (IU/L) & $15.0(12.0-20.0)[0.4-202.0]$ & $16.0(12.0-31.0)[0.3-88.0]$ & $0.772^{\mathrm{c}}$ \\
\hline $\mathrm{HB}(\mathrm{g} / \mathrm{dL})$ & $13.7 \pm 1.9$ & $13.8 \pm 2.3$ & $0.725^{\mathrm{b}}$ \\
\hline $\mathrm{WBC}(/ \mu \mathrm{L})$ & $8.2(6.5-10.2)[2.6-31.9]$ & $9.2(7.3-12.0)[4.4-65.9]$ & $\mathbf{0 . 0 2 8}{ }^{\mathrm{c}}$ \\
\hline $\operatorname{PLT}(/ \mu \mathrm{L})$ & $248.5(203.8-287.0)[17.0-513.0]$ & $248.5(208.5-281.8)$ [31.0-394.0] & $0.987^{c}$ \\
\hline Time to admission (minutes) & $250.0(150.0-480.0)$ [20.0-1.159.0] & $180.0(60.0-350.0)[60.0-880.0]$ & $\mathbf{0 . 0 0 3}^{\mathrm{c}}$ \\
\hline $\mathrm{SAP}(\mathrm{mmHg})$ & $120.0(110.0-132.3)[80.0-220.0]$ & $110.0(100.0-130.0)$ [90.0-200.0] & $\mathbf{0 . 0 3 1} 1^{\mathrm{c}}$ \\
\hline DAP (mmHg) & $70.0(60.0-80.0)[50.0-117.0]$ & $60.0(60.0-80.0)[60.0-100.0]$ & $0.107^{\mathrm{c}}$ \\
\hline Respiratory rate (breaths/min) & $17.0(14.0-20.0)[10.0-26.0]$ & $18.0(16.0-20.0)[14.0-26.0]$ & $0.041^{\mathrm{c}}$ \\
\hline Temperature $\left({ }^{0} \mathrm{C}\right)$ & $36.4(36.1-36.6)[35.5-38.1]$ & $36.4(36.0-36.6)[35.0-38.0]$ & $0.705^{\mathrm{c}}$ \\
\hline Heart rate (bpm) & $84.0(78.0-94.0)[54.0-150.0]$ & $84.0(78.0-100.0)[60.0-157.0]$ & $0.375^{\mathrm{c}}$ \\
\hline $\mathrm{sPO}_{2}(\%)$ & $97.0(95.0-99.0)[80.0-100.0]$ & $97.0(93.0-99.0)[60.0-100.0]$ & $0.672^{\mathrm{c}}$ \\
\hline GCS & $15.0(15.0-15.0)[15.0-15.0]$ & $15.0(15.0-15.0)[9.0-15.0]$ & $<0.001^{\mathrm{c}}$ \\
\hline
\end{tabular}

NC: neurological complication, ${ }^{a}$ : Chi-square test, ${ }^{b}$ : Independent samples $t$ test, ${ }^{c}:$ Mann-Whitney $\mathrm{U}$ test, descriptive statistics are given as $\mathrm{n}(\%)$, mean \pm standard deviation, or median (interquartile range, Q1-Q3) [min-max], as appropriate, COHb: carboxyhemoglobin, BE: base excess, HCO3: bicarbonate, TROP: Troponin, CK: creatine kinase, CKMB: creatine kinase myocardial band, HB: hemoglobin, WBC: white blood cell count, PLT: platelet count, SAP: systolic arterial pressure, DAP: diastolic arterial pressure, sPO2: oxygen saturation, GCS: Glasgow coma scale 
Table 3. Comparison of the patient characteristics according to hyperbaric oxygen therapy status

\begin{tabular}{|c|c|c|c|}
\hline & Without HBO $(n=276)$ & With HBO $(n=16)$ & $\mathbf{p}$ \\
\hline \multicolumn{4}{|l|}{ Gender } \\
\hline Female & $179(64.9 \%)$ & $10(62.5 \%)$ & \multirow{2}{*}{$0.999^{\mathrm{a}}$} \\
\hline Male & $97(35.1 \%)$ & $6(37.5 \%)$ & \\
\hline \multicolumn{4}{|l|}{ Cause of poisoning } \\
\hline Coal stove & $118(42.8 \%)$ & $11(68.8 \%)$ & \multirow{3}{*}{$0.078^{\mathrm{a}}$} \\
\hline Water heater & $40(14.5 \%)$ & $0(0.0 \%)$ & \\
\hline Boiler & $118(42.8 \%)$ & $5(31.3 \%)$ & \\
\hline Age (years) & $42.4 \pm 16.839 .0(30.0-53.0)$ & $45.0 \pm 18.046 .5(32.8-57.8)$ & $0.550^{\mathrm{b}}$ \\
\hline $\mathrm{pH}$ & $7.4(7.4-7.4)[7.1-7.6]$ & 7.4 (7.2-7.4) [6.9-7.5] & $0.056^{\mathrm{c}}$ \\
\hline $\mathrm{COHb}(\%)$ & $20.4 \pm 10.7$ & $28.4 \pm 12.0$ & $0.004^{b}$ \\
\hline Lactate (mmol/L) & $1.9(1.4-2.6)[0.2-13.7]$ & $2.7(1.8-5.4)[0.7-12.3]$ & $0.005^{c}$ \\
\hline $\mathrm{BE}(\mathrm{mmol} / \mathrm{L})$ & $-0.42 \pm 2.80$ & $-5.78 \pm 9.19$ & $\mathbf{0 . 0 3 4}{ }^{\mathrm{b}}$ \\
\hline $\mathrm{HCO}_{3}(\mathrm{mmol} / \mathrm{L})$ & $23.6 \pm 2.6$ & $19.7 \pm 6.6$ & $\mathbf{0 . 0 3 2}^{\mathrm{b}}$ \\
\hline TROP $(\mu \mathrm{g} / \mathrm{L})$ & $0.0(0.0-0.0)[0.0-12.0]$ & $0.1(0.0-0.2)[0.0-4.7]$ & $0.121^{\mathrm{c}}$ \\
\hline $\mathrm{CK}(\mathrm{u} / \mathrm{L})$ & $89.0(61.0-138.0)[0.6-1400.0]$ & $80.0(70.2-122.0)[65.0-1314.0]$ & $0.828^{c}$ \\
\hline CKMB (IU/L) & $15.0(12.0-20.0)[0.3-202.0]$ & $22.0(17.0-32.0)[2.3-88.0]$ & $0.040^{c}$ \\
\hline $\mathrm{HB}(\mathrm{g} / \mathrm{dL})$ & $13.7 \pm 1.9$ & $14.6 \pm 2.5$ & $0.125^{\mathrm{b}}$ \\
\hline $\mathrm{WBC}(/ \mu \mathrm{L})$ & $8.4(6.7-10.4)[2.6-65.9]$ & $10.4(6.5-13.5)[5.5-35.0]$ & $0.314^{\mathrm{c}}$ \\
\hline $\operatorname{PLT}(/ \mu \mathrm{L})$ & $248.5(206.3-285.3)$ [17.0-513.0] & $253.5(185.8-344.8)[138.0-371.0]$ & $0.612^{\mathrm{c}}$ \\
\hline Time to admission (minutes) & $250.0(150.0-480.0)$ [20.0-1159.0] & $120.0(60.0-240.0)[60.0-850.0]$ & $\mathbf{0 . 0 0 3}^{\mathrm{c}}$ \\
\hline $\mathrm{SAP}(\mathrm{mmHg})$ & $120.0(110.0-133.5)$ [80.0-220.0] & $120.0(100.0-130.0)[90.0-180.0]$ & $0.312^{\mathrm{c}}$ \\
\hline $\mathrm{DAP}(\mathrm{mmHg})$ & $70.0(60.0-80.0)$ [50.0-117.0] & $60.0(60.0-75.0)$ [60.0-90.0] & $0.051^{\mathrm{c}}$ \\
\hline Respiratory rate (breaths/min) & $18.0(14.0-20.0)[10.0-26.0]$ & $20.0(16.0-20.0)[14.0-26.0]$ & $0.120^{\mathrm{c}}$ \\
\hline Temperature $\left({ }^{0} \mathrm{C}\right)$ & $36.4(36.1-36.6)[35.5-38.1]$ & $36.2(36.0-36.6)[35.0-37.0]$ & $0.185^{\mathrm{c}}$ \\
\hline Heart rate (bpm) & $84.0(78.0-95.0)[54.0-152.0]$ & $88.0(79.0-100.0)[60.0-157.0]$ & $0.498^{c}$ \\
\hline $\mathrm{sPO}_{2}(\%)$ & $97.0(95.0-99.0)[80.0-100.0]$ & 96.0 (92.0-98.0) [60.0-99.0] & $0.156^{\mathrm{c}}$ \\
\hline GCS & $15.0(15.0-15.0)[9.0-15.0]$ & $15.0(15.0-15.0)[11.0-15.0]$ & $0.003^{c}$ \\
\hline
\end{tabular}

HBO: hyperbaric oxygen, ${ }^{a}$ : Chi-square test, ${ }^{b}$ : Independent samples $t$ test, ${ }^{c}$ : Mann-Whitney $\mathrm{U}$ test, descriptive statistics are given as $\mathrm{n}(\%)$, mean \pm standard deviation, or median (interquartile range, Q1-Q3) [min-max], as appropriate, COHb: carboxyhemoglobin, BE: base excess, HCO3: bicarbonate, TROP: Troponin, CK: creatine kinase, CKMB: creatine kinase myocardial band, HB: hemoglobin, WBC: white blood cell count, PLT: platelet count, SAP: systolic arterial pressure, DAP: diastolic arterial pressure, SPO2: oxygen saturation, GCS: Glasgow coma scale

Table 4. Correlations between the biochemical parameters

\begin{tabular}{|c|c|c|c|c|c|c|}
\hline & \multicolumn{2}{|c|}{ COHb (\%) } & \multicolumn{2}{|c|}{ Lactate $(\mathrm{mmol} / \mathrm{L})$} & \multicolumn{2}{|c|}{ TROP $(\mu \mathrm{g} / \mathrm{L})$} \\
\hline & $\mathbf{r}$ & p & $\mathbf{r}$ & $\mathbf{p}$ & $\mathbf{r}$ & $\mathbf{p}$ \\
\hline $\mathrm{pH}$ & -0.164 & $\mathbf{0 . 0 0 5}^{\mathrm{a}}$ & -0.367 & $<0.001^{a}$ & -0.031 & $0.621^{\mathrm{a}}$ \\
\hline $\mathrm{BE}(\mathrm{mmol} / \mathrm{L})$ & -0.234 & $<0.001^{\text {a }}$ & -0.554 & $<0.001^{\text {a }}$ & -0.154 & $0.014^{\mathrm{a}}$ \\
\hline $\mathrm{HCO}_{3}(\mathrm{mmol} / \mathrm{L})$ & -0.275 & $<0.001^{\text {a }}$ & -0.544 & $<0.001^{a}$ & -0.138 & $0.027^{a}$ \\
\hline $\mathrm{CK}(\mathrm{u} / \mathrm{L})$ & 0.000 & $0.996^{\mathrm{b}}$ & 0.149 & $0.027^{\mathrm{a}}$ & 0.179 & $0.008^{\mathrm{a}}$ \\
\hline CKMB (IU/L) & 0.034 & $0.605^{\mathrm{b}}$ & 0.145 & $0.029^{\mathrm{a}}$ & 0.273 & $<0.001^{\mathrm{a}}$ \\
\hline $\mathrm{HB}(\mathrm{g} / \mathrm{dL})$ & 0.151 & $0.014^{\mathrm{a}}$ & 0.153 & $\mathbf{0 . 0 1 3}^{\mathrm{a}}$ & -0.003 & $0.960^{\mathrm{a}}$ \\
\hline $\mathrm{WBC}(/ \mu \mathrm{L})$ & 0.039 & $0.523^{b}$ & 0.219 & $<0.001^{\text {a }}$ & 0.133 & $0.034^{\mathrm{a}}$ \\
\hline $\operatorname{PLT}(/ \mu \mathrm{L})$ & -0.006 & $0.926^{\mathrm{b}}$ & 0.153 & $\mathbf{0 . 0 1 3}^{\mathrm{a}}$ & 0.123 & $0.049^{a}$ \\
\hline $\mathrm{COHb}(\%)$ & & & 0.369 & $<0.001^{\mathrm{a}}$ & 0.086 & $0.170^{\mathrm{a}}$ \\
\hline Lactate $(\mathrm{mmol} / \mathrm{L})$ & 0.369 & $<0.001^{\text {a }}$ & & & 0.218 & $<0.001^{\mathrm{a}}$ \\
\hline TROP $(\mu \mathrm{g} / \mathrm{L})$ & 0.086 & $0.170^{\mathrm{b}}$ & 0.218 & $<0.001^{a}$ & & \\
\hline
\end{tabular}

CKMB: creatine kinase myocardial band, HB: hemoglobin, WBC: white blood cell count, PLT: platelet count

Table 5. ROC curve analysis of neurological complications

\begin{tabular}{lccccccc}
\hline & AUC & Cut-Off & Sensitivity & Specificity & 95\% CI & Youden's J & p \\
\hline COHb $(\%)$ & 0.815 & $>21.2$ & 88.7 & 56.1 & $0.766-0.858$ & 0.447 & $\mathbf{0 . 0 0 1}$ \\
Lactate $(\mathrm{mmol} / \mathrm{L})$ & 0.629 & $>2.48$ & 50.9 & 75.4 & $0.571-0.685$ & 0.264 & $\mathbf{0 . 0 0 6}$ \\
\hline
\end{tabular}

ROC: receiver operating characteristic, AUC: area under curve, CI: confidence interval, COHb: carboxyhemoglobin

Table 6. ROC curve analysis of hyperbaric oxygen therapy

\begin{tabular}{lccccccc}
\hline & AUC & Cut-Off & Sensitivity & Specificity & 95\% CI & Youden's J & p \\
\hline $\mathrm{COHb}(\%)$ & 0.682 & $>21.8$ & 75.0 & 55.6 & $0.625-0.735$ & 0.306 & $\mathbf{0 . 0 1 4}$ \\
Lactate (mmol/L) & 0.710 & $>2.54$ & 62.5 & 74.7 & $0.654-0.762$ & 0.372 & $\mathbf{0 . 0 0 4}$ \\
\hline
\end{tabular}

ROC: receiver operating characteristic, AUC: area under curve, CI: confidence interval, COHb: carboxyhemoglobin 


\section{DISCUSSION}

The primary aim of this study was to compare and analyze blood lactate and $\mathrm{COHb}$ levels in patients with $\mathrm{CO}$ intoxication so as to identify their use in the interpretation of clinical presentation, the treatment of patients, and their prognoses. A statistically significant difference was found between the blood $\mathrm{CO}$ and lactate levels of intoxicated patients who did and did not develop neurological and cardiac complications. Moreover, a statistically significant difference was found in $\mathrm{COHb}$ and lactate levels among patients who did or did not receive HBO therapy and who were hospitalized or not. Looking at the biochemical profile, a significant correlation was found between $\mathrm{COHb}$ and $\mathrm{pH}$, base excess, and bicarbonate and lactate levels.

The rapid binding of $\mathrm{CO}$ to hemoglobin results in reduced oxygen-carrying capacity of blood, leading subsequently to tissue hypoxia. Due to their high oxygen demand, the brain, heart, and kidneys are the most susceptible organs to the hypoxic effects of $\mathrm{CO}$. Central nervous system involvement is responsible for most symptoms of $\mathrm{CO}$ poisoning. The basis for treatment of $\mathrm{CO}$ intoxication is to administer oxygen which will competitively bind and remove $\mathrm{CO}$ from hemoglobin. This can be achieved by increasing the oxygen concentration or the pressure of inspired air.

Concerning the treatment of $\mathrm{CO}$ intoxication, normobaric and $\mathrm{HBO}$ therapies have an important role in its management. However, there is still debate over their efficacy and applications in different patient groups (1518). That said, specific objective criteria regarding the administration of HBO therapy for $\mathrm{CO}$ poisoning are yet to be defined. Decision to treat is usually based on the clinical findings of patients. Loss of consciousness, signs of neurological involvement, evidence of cardiac ischemia, and severe acidosis are considered indications for HBO therapy (19).

Some studies have reported that the average age of adults admitted to the emergency department with $\mathrm{CO}$ poisoning ranged from 27 to 41 years, and that most patients are female $(6,20)$. The results of this study showed similar findings, where the majority of $\mathrm{CO}$ poisoning patients were female, and the mean age was $42.5 \pm 16.9$ years.

In this study, $\mathrm{COHb}$ and lactate levels were significantly higher in patients presenting with neurological symptoms. Solkal et al. (25) reported that patients with severe $\mathrm{CO}$ poisoning had significantly higher lactate levels when compared to patients with mild poisoning. Özkan et al. (21) indicated that lactate and $\mathrm{COHb}$ were significantly elevated in patients with $\mathrm{CO}$ poisoning. A study by Aslan et al. (22) similarly associated elevated $\mathrm{COHb}$ levels with an increased likelihood of developing neurological symptoms.

In a small series, Inoue et al. (13) demonstrated that blood lactate levels were a useful predictor of prognosis among patients who sustained severe $\mathrm{CO}$ poisoning due to attempted suicide. Furthermore, Moon et al. (12) studied 80 patients who presented to the emergency department with $\mathrm{CO}$ poisoning and found that their initial blood lactate level was an independent predictor of severe complications and intensive care unit admission. It was found herein that lactate levels could predict hospital admissions and cardiac and neurological complications. These results were consistent with the literature.
In the present study, a strong and significant correlation was found between $\mathrm{COHb}$ and $\mathrm{pH}$, base excess, bicarbonate, and lactate levels. Moreover, lactate levels were strongly and significantly correlated with $\mathrm{COHb}$, troponin, $\mathrm{pH}$, base excess, and bicarbonate levels. Besli et al. (24) reported a positive correlation between lactate and $\mathrm{COHb}$ levels, as well as elevated lactate levels in patients who developed neurological symptoms. A recent study by Dogan et al. (7) found that the initial lactate levels of 74 patients with severe $\mathrm{CO}$ poisoning were positively correlated with $\mathrm{COHb}$ levels.

The results of this study showed that patients with elevated $\mathrm{COHb}$ and lactate levels were significantly more likely to develop neurological complications. Benaissa et al. (14) found that lactate levels were found to be significantly higher in intoxicated patients who developed neurological symptoms, but concluded that the clinical significance of this finding was questionable since the increase in lactate levels were minimal.

Also a result of this study, lactate levels of patients with cardiac findings were significantly higher. Similarly, Cervellin et al. (26) and Marchewka et al. (27) found that lactate levels were a useful predictor in determining the severity of $\mathrm{CO}$ intoxication, and were also well correlated with troponin levels.

Repplinger et al. (23) argued that lactate could be used as an indicator for HBO therapy. It was similarly found herein that $\mathrm{COHb}$ and lactate levels increased in correlation and also that patients with high lactate levels were more likely to be hospitalized. However, since lactate levels are influenced by multiple factors, further studies are needed for more conclusive results.

\section{CONCLUSION}

This study shows that $\mathrm{COHb}$ and lactate levels acquired at admission are significant in the prognosis, follow-up, and treatment of patients admitted to the emergency department with $\mathrm{CO}$ poisoning. Elevated lactate and $\mathrm{CO}$ levels may be associated with neurological and cardiac complications.

Ethics Committee Approval: The study was approved by the Ethics Committee of Dışkapı Yıldırım Beyazıt, Training and Research Hospital (16.02.2015, 20/04).

Conflict of Interest: None declared by the authors.

Financial Disclosure: None declared by the authors.

Acknowledgements: None declared by the authors.

Author Contributions: Idea/Concept: MHH; Design: EU; Data Collection/Processing: MHH; Analysis/Interpretation: AH; Literature Review: $\mathrm{AH}$; Drafting/Writing: EU; Critical Review: EU, AH, MHH.

\section{REFERENCES}

1. Ernst A, Zibrak JD. Carbon monoxide poisoning. $\mathrm{N}$ Engl J Med. 1998;339(22):1603-8.

2. Weaver LK. Clinical practice. Carbon monoxide poisoning. N Engl J Med. 2009;360(12):1217-25. 
3. Thom SR. Carbon monoxide pathophsyology and treatment. In: Neuman TS, Thom SR, eds. Phsyology and medicine of hyperbaric oxygen therapy. Philadelphia: Saunders Elsevier; 2008. p.321-47.

4. Crocker PJ, Walker JS. Pediatric carbon monoxide toxicity. J Emerg Med. 1985;3(6):443-8.

5. Graber JM, Smith AE. Results from a state-based surveillance system for carbon monoxide poisoning. Public Health Rep. 2007;122(2):145-54.

6. Keleş A, Demircan A, Kurtoğlu G. Carbon monoxide poisoning: how many patients do we miss? Eur J Emerg Med. 2008;15(3):154-7.

7. Doğan NÖ, Savrun A, Levent S, Günaydın GP, Çelik GK, Akküçük H, et al. Can initial lactate levels predict the severity of unintentional carbon monoxide poisoning? Hum Exp Toxicol. 2015;34(3):324-9.

8. Buckley NA, Juurlink DN, Isbister G, Bennett MH, Lavonas EJ. Hyperbaric oxygen for carbon monoxide poisoning. Cochrane Database Syst Rev. 2011;4:CD002041.

9. Türkdoğan KA. Pratik acil tıp. İstanbul: EMA; 2020.

10. Huang CC, Chung MH, Weng SF, Chien CC, Lin SJ, Lin HJ, et al. Long-term prognosis of patients with carbon monoxide poisoning: a nationwide cohort study. PLoS One. 2014;9(8):e105503.

11. Zou JF, Guo Q, Shao H, Li B, Du Y, Liu M, et al. A positive Babinski reflex predicts delayed neuropsychiatric sequelae in Chinese patients with carbon monoxide poisoning. Biomed Res Int. 2014;2014:814736.

12. Moon JM, Shin MH, Chun BJ. The value of initial lactate in patients with carbon monoxide intoxication: in the emergency department. Hum Exp Toxicol. 2011;30(8):836-43.

13. Inoue S, Saito T, Tsuji T, Tamura K, Ohama S, Morita $\mathrm{S}$, et al. Lactate as a prognostic factor in carbon monoxide poisoning: a case report. Am J Emerg Med. 2008;26(8):966.e1-3.

14. Benaissa ML, Mégarbane B, Borron SW, Baud FJ. Is elevated plasma lactate a useful marker in the evaluation of pure carbon monoxide poisoning? Intensive Care Med. 2003;29(8):1372-5.

15. Wolf SJ, Lavonas EJ, Sloan EP, Jagoda AS. Clinical policy: critical issues in the management of adult patients presenting to the emergency department with acute carbon monoxide poisoning. Ann Emerg Med. 2008;51(2):138-52.
16. Annane D, Chadda K, Gajdos P, Jars-Guincestre MC, Chevret S, Raphael JC. Hyperbaric oxygen therapy for acute domestic carbon monoxide poisoning: two randomized controlled trials. Intens Care Med. 2011;37(3):486-92.

17. Weaver LK, Valentine KJ, Hopkins RO. Carbon monoxide poisoning: risk factors for cognitive sequelae and the role of hyperbaric oxygen. Am J Respir Crit Care Med. 2007;176(5):491-7.

18. Sanders RW, Katz KD, Suyama J, Akhtar J, O'Toole KS, Corll D, et al. Seizure during hyperbaric oxygen therapy for carbon monoxide toxicity: a case series and five-year experience. J Emerg Med. 2012;42(4): e69-72.

19. Kandiş H, Katırcı Y, Karapolat BS. Carbon monoxide poisoning. Duzce Med J. 2009;11(3):54-60.

20. Sá MC, Rodrigues RP, Moura D. Carbon monoxide intoxications in Portugal. Acta Med Port. 2011;24(5):727-34.

21. Ozkan S, Salt O, Durukan P, Sen A, Bulbul E, Duman A, et al. The relationship among plasma copeptin, carboxyhemoglobin, and lactate levels in carbon monoxide poisoning. Hum Exp Toxicol. 2020;39(3):311-8.

22. Aslan S, Erol MK, Karcioglu Ö, Meral M, Cakir Z, Katirci Y. The investigation of ischemic myocardial damage in patients with carbon monoxide poisoning. Anadolu Kardiyol Derg. 2005;5(3):189-93.

23. Repplinger DJ, Slomer A, Nolan B, Su MK. Lactate and carbon monoxide poisoning: More questions regarding its prognostic role. Hum Exp Toxicol. 2016;35(7):794.

24. Besli GE, Ergüven M, Karadoğan M, Yılmaz O. Carbon monoxide poisoning in children. Eurasian $\mathbf{J}$ Emerg Med. 2010;9(1):26-30.

25. Sokal JA, Kralkowska E. The relationship between exposure duration, carboxyhemoglobin, blood glucose, pyruvate and lactate and the severity of intoxication in 39 cases of acute carbon monoxide poisoning in man. Arch Toxicol. 1985;57(3):196-9.

26. Cervellin G, Comelli I, Rastelli G, Picanza A, Lippi G. Initial blood lactate correlates with carboxyhemoglobin and clinical severity in carbon monoxide poisoned patients. Clin Biochem. 2014;47(18):298-301.

27. Marchewka J, Gawlik I, Dębski G, Popiołek L, Marchewka W, Hydzik P. Cardiological aspects of carbon monoxide poisoning. Folia Med Cracov. 2017;57(1):75-85. 